\title{
Ptychographic measurements of ultrahigh-intensity laser-plasma interactions
}

\author{
A. Leblanc ${ }^{1}$, S. Monchocé', C. Bourassin-Bouchet ${ }^{2}$, S. Kahaly ${ }^{1 \uparrow}$ and F. Quéré ${ }^{1 \star}$
}

The extreme intensities now delivered by femtosecond lasers make it possible to drive and control relativistic motion of charged particles with light ${ }^{1}$, opening a path to compact particle accelerators $^{2,3}$ and coherent X-ray sources ${ }^{4,5}$. Accurately characterizing the dynamics of ultrahigh-intensity laser-plasma interactions as well as the resulting light and particle emissions is an essential step towards such achievements. This remains a considerable challenge, as the relevant scales typically range from picoseconds to attoseconds in time, and from micrometres to nanometres in space. In these experiments, owing to the extreme prevalent physical conditions, measurements can be performed only at macroscopic distances from the targets, yielding only partial information at these microscopic scales. This letter presents a major advance by applying the concepts of ptychography ${ }^{6,7}$ to such measurements, and thus retrieving microscopic information hardly accessible until now. This paves the way to a general approach for the metrology of extreme laser-plasma interactions on very small spatial and temporal scales.

Ptychography is a general lensless imaging technique, used with both light ${ }^{6,7}$ and electron beams ${ }^{8}$, to reconstruct images with simultaneous amplitude and phase contrast. It consists in measuring the intensity of the diffraction pattern produced by a probe beam diffracted out by an object to be imaged, typically placed at or around the beam focus, for different relative positions $\mathbf{r}_{0}$ between the probe and object. With $E(\mathbf{r})$ the complex field of the probe in the object plane, and $O(\mathbf{r})$ the complex transmission of the object at the probe wavelength (where $\mathbf{r}$ is the position vector transverse to the propagation direction), the obtained data set is described mathematically as:

$$
I\left(\mathbf{k}, \mathbf{r}_{0}\right)=\left|\iint E(\mathbf{r}) O\left(\mathbf{r}-\mathbf{r}_{0}\right) \mathrm{e}^{i \mathbf{k} \cdot \mathbf{r}} \mathrm{d}^{2} \mathbf{r}\right|^{2}
$$

with $\mathbf{k}$ the two-dimensional (2D) wavevector component transverse to the propagation direction. Iterative phase-retrieval algorithms can then be applied to this data set to reconstruct not only the object $O(\mathbf{r})$, but also the probe field $E(\mathbf{r})($ refs 7,9$)$. In this letter, we present a conceptual advance by showing that the general principles of ptychography can be adapted to ultrahigh-intensity laser-plasma interactions, up to the relativistic regime, to obtain microscopic physical quantities that would be extremely difficult to measure by other means. Using this new measurement scheme, we simultaneously retrieve the complex field spatial profile, in the target plane, of coherent extreme-ultraviolet light beams emitted by laser-driven plasmas (probe field $E(\mathbf{r}))$ in different interaction regimes, and the spatial profile of a microstructured plasma surface (object $O(\mathbf{r})$ ).

We apply this measurement technique to dense plasmas generated by ultrahigh-intensity femtosecond laser pulses focused at the surface of initially solid targets. Owing to their high density and sharp interface with the vacuum, such plasmas specularly reflect the incident laser beam, and are therefore called plasma mirrors ${ }^{10}$. At ultrahigh light intensities $\left(>10^{16} \mathrm{~W} \mathrm{~cm}^{-2}\right)$, the temporal response of these mirrors to the incident field becomes nonlinear, leading through different mechanisms to the generation in the reflected beam of trains of attosecond pulses, associated in the frequency domain with combs of high-order harmonics of the laser frequency ${ }^{11,12}$, which can extend up to the X-ray range ${ }^{13,14}$. This scheme is considered as one of the most promising paths to obtain intense attosecond light sources for ultrafast science ${ }^{15-17}$.

Spatially, each harmonic is described by a complex field $h_{n}(\mathbf{r})=\left|h_{n}(\mathbf{r})\right| \mathrm{e}^{i \varphi_{n}(\mathbf{r})}$, where $\mathbf{r}$ is the transverse spatial position in the target plane and $n$ the harmonic order. Owing to the nonlinearity of the generation process, the amplitude $\left|h_{n}(\mathbf{r})\right|$ is expected to cover a fraction of the laser focal spot, and its size is thus typically in the micrometre range. As far as the harmonic phase $\varphi_{n}$ is concerned, it typically varies with the driving laser intensity $I_{\mathrm{L}}$, whatever the generation process ${ }^{18,19}$. The spatial variation $I_{\mathrm{L}}(\mathbf{r})$ of this intensity at focus therefore leads to a spatially curved harmonic phase $\varphi_{n}(\mathbf{r})$, which can strongly affect the divergence of the produced harmonic beam. Accurately determining these amplitude and phase profiles is essential for further applications of these light sources, and is precisely one of the outcomes of our ptychographic measurements.

The key idea is to generate the harmonics on a spatially modulated plasma mirror surface, which acts as the object $O(\mathbf{r})$ by introducing a small phase perturbation on the harmonic beam $h_{n}(\mathbf{r})$ (Fig. 1). This harmonic beam constitutes the probe $E(\mathbf{r})$, which is here directly generated on the object, instead of being provided by an external source as in usual ptychography.

Experimentally, the modulated plasma mirror surface is obtained in situ by optically microstructuring an initially flat solid target ${ }^{20}$. As illustrated in Fig. 1a, two non-collinear prepulses (dark red) ionize the target at a delay $\tau$ before the ultrahigh-intensity pulse which generates the harmonics. Their spatial interference pattern modulates the prepulse fluence along one spatial dimension $x$ (Fig. 1b,c, grey scale), leading to a corresponding modulation of the expansion velocity of the plasma into the vacuum. A spatial modulation $g(x)$ of the plasma surface position then grows from the initially flat surface (Fig. 1d), in the time interval $\tau$ between target ionization and the arrival of the main laser pulse. These

\footnotetext{
${ }^{1}$ Lasers, Interactions and Dynamics Laboratory (LIDyL), Commissariat à I'Energie Atomique, Université Paris-Saclay, DSM/IRAMIS, CEN Saclay, 91191 Gif sur Yvette, France. ${ }^{2}$ Synchrotron SOLEIL, Université Paris-Saclay, Saint-Aubin, BP 34, 91192 Gif-sur-Yvette, France. ${ }^{\dagger}$ Present address: ELI-ALPS, ELI-Hu Nkft, Dugonics ter 13, Szeged 6720, Hungary. *e-mail: fabien.quere@cea.fr
} 

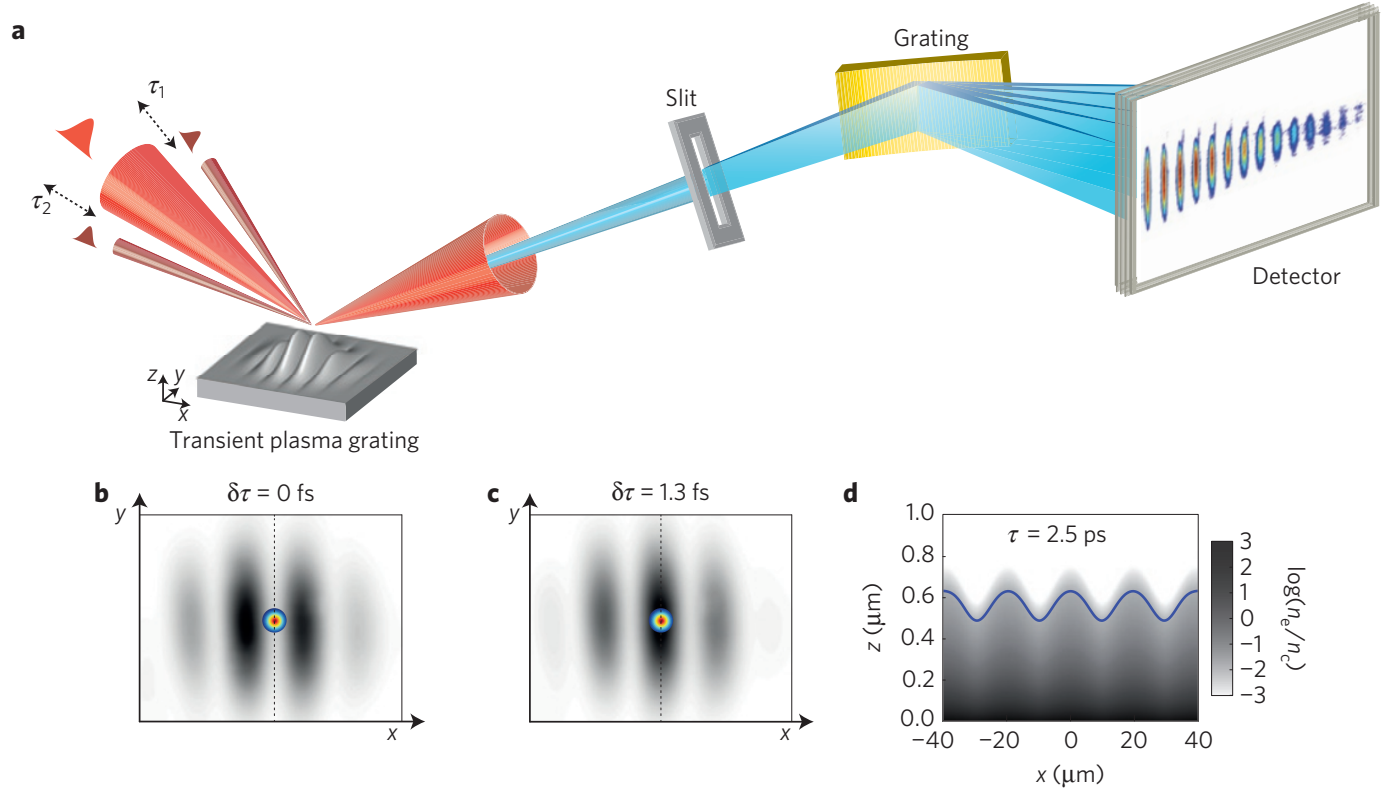

Figure 1| Measurement scheme. a-d, Sketch of the experiment (a). Two prepulse beams $\left(I \approx 10^{16} \mathrm{~W} \mathrm{~cm}^{-2}\right)$ cross at the surface of an initially solid target, which they strongly ionize. This initiates a plasma expansion with a velocity modulated in space owing to the prepulse interference (grey images in $\mathbf{b}$ and c). This leads to the growth of a transient plasma surface modulation, as illustrated by the hydrodynamic simulations ${ }^{20,21}$ shown in $\mathbf{d}$, for a delay $\tau=2.5 \mathrm{ps}$ after the prepulses (grey scale: electron density $n_{e}$, blue curve: isodensity surface $g(x)$ corresponding to the critical plasma density $n_{c}$ ). An ultrahigh-intensity main laser pulse ( $25 \mathrm{fs}$ pulse centred at $800 \mathrm{~nm}$, intensity up to $7 \times 10^{18} \mathrm{~W} \mathrm{~cm}^{-2}$, focal spot shown by the coloured spots in $\mathbf{b}$ and $\mathbf{c}$ ) then interacts with this modulated plasma, and generates a beam of high-order harmonics in the specular direction. This harmonic beam is spectrally resolved with a 2D spectrometer that measures for each harmonic the 1D diffraction pattern along the direction of surface modulation. This spectrally resolved diffraction profile is measured as a function of the relative position $x_{0}$ between the surface modulation and the main pulse focus, scanned by changing the delay $\delta \tau=\tau_{2}-\tau_{1}$ between the two prepulse beams by fractions of the laser period, leading to a shift of the interference fringes (compare $\mathbf{b}$ and c). All experiments were performed with UHI100, the 100-TW, 25-fs Ti-Sa laser at CEA Saclay. See Supplementary Methods for more information.

so-called transient plasma gratings evolve on a timescale of hundreds of femtoseconds to picoseconds (typical expansion velocity of $10^{-4} c=30 \mathrm{~nm} \mathrm{ps}^{-1}$ ), and thus appear as static objects to the 25-fs-long main laser pulse. We spectrally resolve the harmonic beam produced by the main pulse on this plasma grating, and measure the $1 \mathrm{D}$ diffraction pattern of each harmonic along the direction of the surface modulation (Fig. 1a).

For a relative position $x_{0}$ between this shaped surface and the laser focal spot that generates the harmonics (Fig. 1b,c, colour scale), a phase modulation $\mathrm{e}^{i \phi_{n}\left(x-x_{0}\right)}$ is induced on the harmonic beam $h_{n}(x)$, where $\phi_{n}\left(x-x_{0}\right)=2 \pi \cos \theta g\left(x-x_{0}\right) / \lambda_{n}$, with $\lambda_{n}$ the harmonic wavelength and $\theta$ the laser incidence angle on target. After diffraction, taking into account the geometry of the spectrally and angularly resolved measurement system, the beam profile of the $n$th harmonic in the detector plane is given by (see Supplementary Methods):

$$
I_{n}\left(k_{x}, x_{0}\right)=\left|\int h_{n}(x) \mathrm{e}^{i \phi_{n}\left(x-x_{0}\right)} \mathrm{e}^{i k_{x} \cdot x} \mathrm{~d} x\right|^{2}
$$

This is the $1 \mathrm{D}$ equivalent of the generic equation for ptychography, equation (1), with a phase-only probe. This suggests that, using one of the algorithms of ptychography, both the complex harmonic field $h_{n}(x)$ and the plasma surface shape $g(x)$ can be retrieved from measurements of the harmonic diffraction pattern as a function of $x_{0}$.

We thus perform our ptychographic measurements using optically induced plasma structures as diffracting objects. In principle, these could also be performed using pre-structured solid targets (for example, grating targets), but this can be demanding on the pointing stability of the laser beam, which then needs to be matched to the spatial scale of the target modulations. In contrast, with the all-optical scheme used here, all beams have the same spatial jitter: the transient plasma grating and the main laser focal spot are thus spatially locked on target. For the ptychographic measurements, the relative position $x_{0}$ of the fringe pattern with respect to the main pulse focal spot is scanned simply by changing the relative delay $\delta \tau=\tau_{2}-\tau_{1}$ between the two prepulse beamlets by small fractions of the laser optical period (Fig. 1b,c).

The spatial period $l_{\mathrm{g}}$ of the surface modulation can be controlled through the lateral distance between the two beamlets before focusing. In the case shown in Fig. 1b,c, we chose this distance such that $l_{\mathrm{g}} \simeq 22 \mu \mathrm{m}$ is larger than the main beam waist $w_{0} \simeq 6.2 \mu \mathrm{m}$ (half-width at $1 / \mathrm{e}$ in amplitude). In these conditions, the harmonics are generated from a slowly modulated plasma surface, whose tilt and curvature depend on the relative position $x_{0}$. Using the set-up described in the Supplementary Methods, we were able to sample this shaped surface with the main laser focal spot with an average spatial step of $\delta x_{0}=220 \mathrm{~nm}$ on $x_{0}$, corresponding to an average time step of 26 attoseconds on $\delta \tau$. For one ptychographic trace, we typically measure more than 100 diffraction patterns for different values of $x_{0}$ covering one full spatial period of the transient plasma grating.

We first performed measurements at the highest intensity achievable with the main beam, $I=7 \times 10^{18} \mathrm{~W} \mathrm{~cm}^{-2}$. In these conditions, the harmonics result from the periodic Doppler shift induced on the reflected field by the laser-driven relativistic oscillation of the plasma surface ${ }^{10}$-the so-called relativistic oscillating mirror (ROM) mechanism. Typical spectrally resolved harmonic diffraction patterns $I_{n}\left(k_{x}, x_{0}\right)$, for harmonic orders 11 to $\sim 25$, are shown in Fig. 2 for four different relative positions $x_{0}$ (see Supplementary Movie 1 for a complete scan). These reveal how information on the harmonic beam and plasma surface modulation is encoded into the measured data. As $x_{0}$ varies, the local tilt of the 

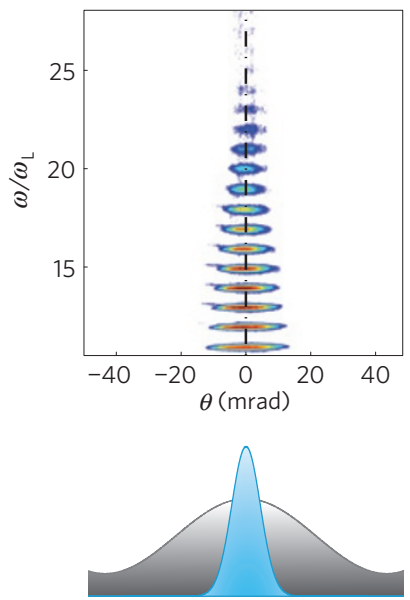
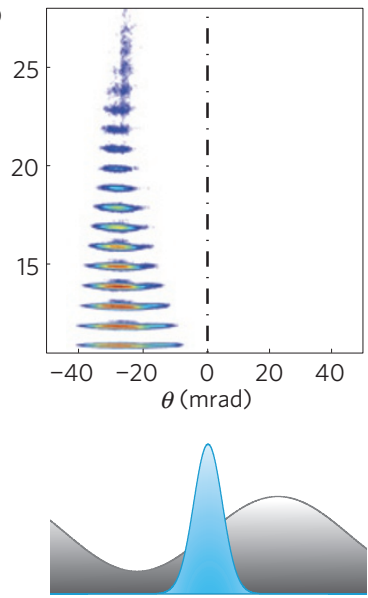
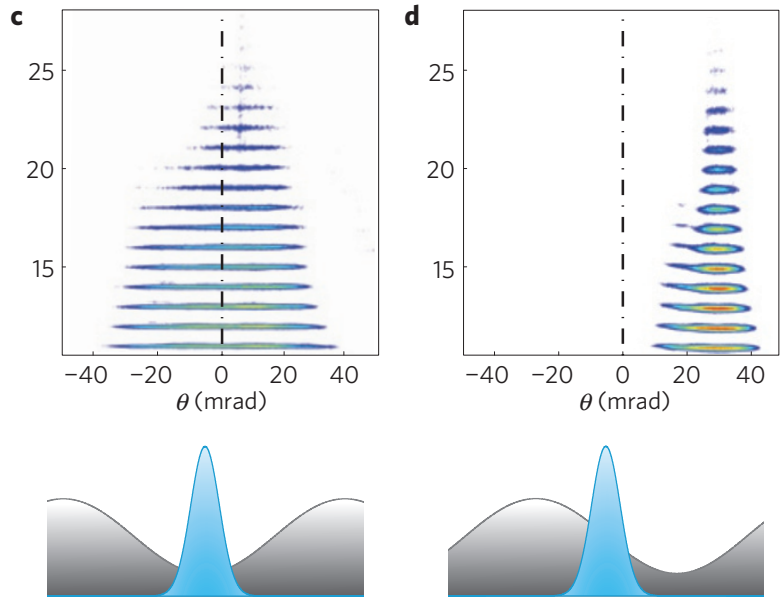

Figure 2 | Harmonic beams generated at different positions on a transient plasma grating. a-d, Four spectrally resolved harmonic beams, generated for different positions $x_{0}$ of the main laser focal spot on the modulated plasma surface, as indicated in the lower panels (harmonic source sketched as the blue Gaussian, rippled surface sketched as the grey area). These harmonics have been generated in the relativistic oscillating mirror (ROM) regime, at a peak intensity of $7 \times 10^{18} \mathrm{~W} \mathrm{~cm}^{-2}$, with a main beam focal spot smaller than the spatial period of the transient grating (see Fig. 1a,b). In $\mathbf{b}$ and $\mathbf{d}$, the surface modulation seen by the harmonic source is quasi-linear, thus mostly leading to a deflection of the harmonic beam. In $\mathbf{a}$ and $\mathbf{c}$, the local surface curvatures lead to changes in beam divergence, as is particularly noticeable in $\mathbf{c}$. These two effects respectively encode information on the plasma surface shape $g(x)$ and on the complex harmonic field $h_{n}(x)$.

shaped plasma surface changes. This leads to the global angular deflection of the harmonic beam observed in the experiment (Fig. 2b,d). The average direction $\theta_{n}\left(x_{0}\right)$ of this beam thus oscillates with $x_{0}$, and this oscillation directly maps the derivative $\mathrm{d} g / \mathrm{d} x$ of the plasma surface shape, as in deflectometry ${ }^{25}$.

In contrast, information on the harmonic source $h_{n}(x)$ is rather encoded in the variations of the divergence $\Delta \theta_{n}\left(x_{0}\right)$ of the harmonic beam, induced by the variable local curvature of the shaped plasma surface (Fig. 2a-c). For a given surface curvature, for obvious geometric reasons, the larger the harmonic source $\left|h_{n}(x)\right|$ in the target plane, the stronger the effect on $\Delta \theta_{n}$. This change in $\Delta \theta_{n}$ also carries information on the phase $\varphi_{n}(x)$ of $h_{n}(x)$. For instance, in Fig. 2, $\Delta \theta_{n}$ strongly increases for a concave plasma surface (compare panel $c$ to panels $b$ and $d$ ), but is slightly reduced when the surface is convex (panel a). This asymmetry reveals that the harmonic field has converging wavefronts in the source plane, which gets compensated by the opposite surface curvature in the case of panel a, whereas their curvature is enhanced in the case of panel c.

Having described how information is encoded into the measured diffraction patterns in this particular measurement regime, we now extract it by using one of the iterative phase-retrieval algorithms developed for ptychography (see Supplementary Methods). For each harmonic order $n$ measured in Fig. 2, this algorithm is separately applied to the ptychographic data set $I_{n}\left(k_{x}, x_{0}\right)$. An example of such a data set is shown in Fig. 3a, for the 12th harmonic generated by the ROM mechanism at a prepulse delay $\tau=2.5 \mathrm{ps}$. The quality of these data, involving more than 100 shots, provides one of the most striking pieces of evidence so far of the possibility of achieving excellent shot-to-shot reproducibility in ultrahigh-intensity laserplasma experiments - a prerequisite for the application of the present measurement technique. The phase-retrieval procedure converges to the reconstructed trace shown in Fig. $3 \mathrm{~b}$.

The amplitude (full line) and phase (dashed line) of the harmonic field $h_{12}(x)$ in the target plane retrieved from this data set are shown in Fig. 3c. Similar reconstructions are obtained for all measured harmonic orders, from a single experimental scan of $x_{0}$. The width of the 12th harmonic source is $2.2 \mu \mathrm{m}$ (half-width at $1 / \mathrm{e}$ in amplitude), corresponding to $35 \%$ of the main laser focal spot size, and like in particle-in-cell simulations ${ }^{18}$, a decrease is observed for increasing harmonic orders. This source exhibits a positive parabolic spatial phase $\varphi_{n}(x)$, corresponding to converging wavefronts in the target plane. Theory shows that this phase results from the deformation $\delta z(x)$ of the plasma surface induced by the spatially varying pressure (typically several Gbar) exerted by the laser field as it reflects, leading to $\varphi_{n}(x)=k_{n} \cos \theta \delta z(x)$ (refs 18,26). These measurements thus provide direct access to the nanometrescale plasma surface deformation induced by the ultrahigh-intensity laser field.

To demonstrate the versatility of this ptychographic measurement scheme, we now apply it to another regime of laser-plasma interactions, involving a totally different mechanism of harmonic generation. To this end, we reduce the peak intensity of the main laser pulse by more than one order of magnitude, down to $3.10^{17} \mathrm{~W} \mathrm{~cm}^{-2}$, by aperturing the main beam before focusing. In this regime, ROM becomes inefficient owing to the non-relativistic velocity of the plasma surface. Harmonics are instead emitted by collective electronic plasma oscillations periodically excited by electron bunches injected in the dense part of the plasma by the incident laser field-a mechanism known as coherent wake emission (CWE; refs 12,27). In addition, we decrease the grating period $l_{\mathrm{g}}$ down to $14.3 \mu \mathrm{m}$, such that it now becomes comparable to the main beam waist $\left(w_{0}=14.5 \mu \mathrm{m}\right)$. In these conditions, the information encoded in the beam diffraction pattern is no longer that previously described for $w_{0} \ll l_{\text {g }}$, and we are thus able to test our ptychographic scheme in a different measurement regime.

Figure $3 \mathrm{~g}$ shows the ptychographic trace measured in this regime, from which we retrieve the complex harmonic field (12th order) shown in Fig. 3e, using the same reconstruction procedure as before. The properties of the harmonic field are now totally different from those measured in the ROM regime. Its amplitude profile has a size that is much closer to the main beam waist $w_{0}$ than in the ROM regime (see upper axis in Fig. $3 \mathrm{c}-\mathrm{e}$ ), owing to the lower intrinsic nonlinearity of the CWE mechanism ${ }^{12,27}$. The spatial phase $\varphi_{12}(x)$ is now negative, corresponding to diverging wavefronts on target, and has a stronger curvature than in the ROM regime. Theory shows that this is due to the totally different physical origin of this phase ${ }^{28}$, which is induced by the variation with laser intensity of the arrival time of the laser-injected electron bunches to the dense part of the plasma $^{28}$. Although such differences between CWE and ROM had been previously observed in simulations and inferred from indirect 


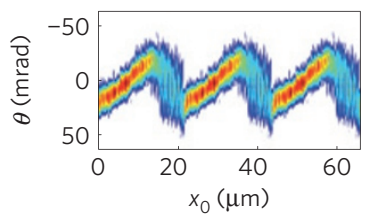

b

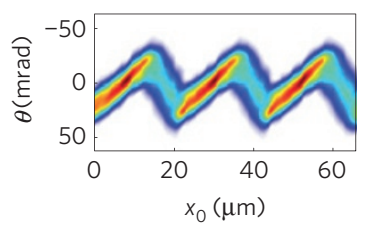

c

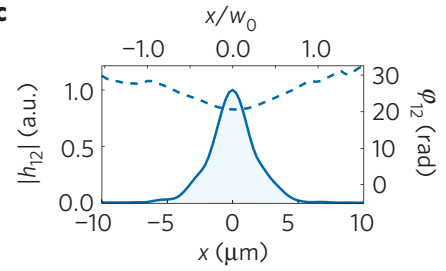

d

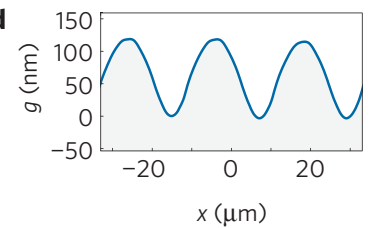

e
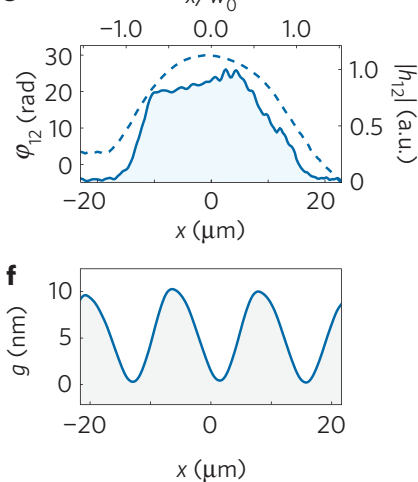

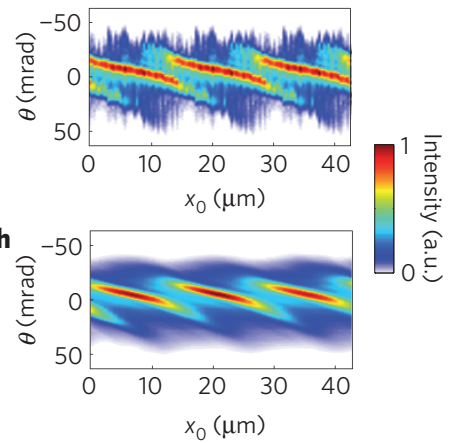

Figure 3 | Ptychographic measurements of different harmonic sources and plasma surface shapes. a,g, Two examples of ptychographic data sets for the

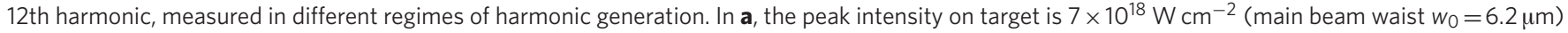
and the prepulse delay $\tau=2.5 \mathrm{ps}$, so that the harmonics are generated by the ROM mechanism. In $\mathbf{g}$, the intensity is reduced to $3 \times 10^{17} \mathrm{~W} \mathrm{~cm}^{-2}$ (main beam waist $w_{0}=14.5 \mu \mathrm{m}$ ) and the prepulse delay to $\tau=0.25 \mathrm{ps}$, and the harmonics are then produced by coherent wake emission 22 . The grating period $l_{\mathrm{g}}$ was also changed between these two measurements $\left(I_{g}=22 \mu \mathrm{m}\right.$ in $\mathbf{a}, 14.3 \mu \mathrm{m}$ in $\left.\mathbf{g}\right)$, to test different measurement regimes. $\mathbf{b}, \mathbf{h}$, Corresponding traces reconstructed by the phase-retrieval algorithm. c,e, Corresponding amplitude (full lines) and phase (dashed lines) profiles of the harmonic source $h_{12}$ ( $x$ ) respectively retrieved in each case. $\mathbf{d}, \mathbf{f}$, Retrieved plasma surface shape $g(x)$. Note that the measurement of a can be qualitatively understood as a spatial analogue of the temporal measurements of attosecond light pulses in the streaking regime 23,24 .

experimental evidence ${ }^{27,28}$, they had never been observed in such a direct way.

In addition to the complex harmonic field, these ptychographic measurements also provide information on the plasma surface profile $g(x)$. The retrieved profiles corresponding to the data sets of Fig. 3a and $g$ are respectively shown in Fig. $3 \mathrm{~d}$ and $\mathrm{f}$, and are oscillating functions with nanometric amplitudes, as expected. The clear change in modulation depth between the two cases shows how the temporal dynamics of this evolving plasma structure can also be resolved, simply by changing the delay $\tau$ between the prepulses and the main pulse. These measurements demonstrate that our experimental scheme can be exploited both to induce spatial structures on initially plain targets, and to measure these structures and their temporal evolution. This is of great interest for high-field science, where structured plasma surfaces provide a promising avenue, for example, for new ion acceleration processes ${ }^{29}$ and ultrahigh-intensity plasmonics ${ }^{30-32}$.

These results show that it is possible to transpose the concepts and tools of ptychography, and more generally of coherent diffraction imaging, to different regimes of ultrahigh-intensity laser-plasma interactions. By adding time resolution to the in situ ptychographic scheme demonstrated here (for example, through the use of rapidly evolving diffracting objects ${ }^{33,34}$ ), extreme lasermatter interactions might eventually be resolved not only in space, but also in time, down to the attosecond timescale. This work thus opens a new conceptual framework for metrology in ultrahighintensity physics, extendable to X-rays and, potentially, particle beams generated by laser-plasma interactions. Such measurements will be essential for the accurate characterization of these unique light and particle sources, and will provide access to microscopic information on ultrahigh-intensity physics that can otherwise be derived only by very indirect means.

Received 28 January 2015; accepted 12 November 2015; published online 14 December 2015

\section{References}

1. Mourou, G. A., Tajima, T. \& Bulanov, S. V. Optics in the relativistic regime. Rev. Mod. Phys. 78, 309-371 (2006).

2. Malka, V. et al. Principles and applications of compact laser-plasma accelerators. Nature Phys. 4, 447-453 (2008)
3. Daido, H., Nishiuchi, M. \& Pirozhkov, A. S. Review of laser-driven ion sources and their applications. Rep. Prog. Phys. 75, 056401 (2012).

4. Sansone, G., Poletto, L. \& Nisoli, M. High-energy attosecond light sources. Nature Photon. 5, 655-663 (2011).

5. Corde, S. et al. Femtosecond x rays from laser-plasma accelerators. Rev. Mod. Phys. 85, 1-48 (2013).

6. Rodenburg, J. M. et al. Hard-x-ray lensless imaging of extended objects. Phys. Rev. Lett. 98, 034801 (2007).

7. Thibault, P. et al. High-resolution scanning $\mathrm{x}$-ray diffraction microscopy. Science 321, 379-382 (2008).

8. Nellist, P. D., Mccallum, B. \& Rodenburg, J. M. Resolution beyond the 'information limit' in transmission electron microscopy. Nature 374, 630-632 (1994).

9. Thibault, P., Dierolf, M., Bunk, O., Menzel, A. \& Pfeiffer, F. Probe retrieval in ptychographic coherent diffractive imaging. Ultramicroscopy 109, 338-343 (2009).

10. Thaury, C. et al. Plasma mirrors for ultrahigh-intensity optics. Nature Phys. 3, 424-429 (2007)

11. Teubner, U. \& Gibbon, P. High-order harmonics from laser-irradiated plasma surfaces. Rev. Mod. Phys. 81, 445-479 (2009).

12. Thaury, C. \& Quéré, F. High-order harmonic and attosecond pulse generation on plasma mirrors: Basic mechanisms. J. Phys. B 43, 213001 (2010).

13. Dromey, B. et al. High harmonic generation in the relativistic limit. Nature Phys. 2, 456-459 (2006)

14. Dromey, B. et al. Bright multi-keV harmonic generation from relativistically oscillating plasma surfaces. Phys. Rev. Lett. 99, 085001 (2007).

15. Tsakiris, G. D., Eidmann, K., Meyer-ter-Vehn, J. \& Krausz, F. Route to intense single attosecond pulses. New J. Phys. 8, 19 (2006).

16. Quéré, F. Ultrafast science: Attosecond plasma optics. Nature Phys. 5, 93-94 (2009).

17. Ma, G. et al. Intense isolated attosecond pulse generation from relativistic laser plasmas using few-cycle laser pulses. Phys. Plasmas 22, 033105 (2015).

18. Vincenti, H., Monchocé, S., Kahaly, S., Martin, P. \& Quéré, F. Optical properties of relativistic plasma mirrors. Nature Commun. 5, 3403 (2014).

19. Malvache, A., Borot, A., Quéré, F. \& Lopez-Martens, R. Coherent wake emission spectroscopy as a probe of steep plasma density profiles. Phys. Rev. E 87, 035101 (2013).

20. Monchocé, S. et al. Optically controlled solid-density transient plasma gratings. Phys. Rev. Lett. 112, 145008 (2014).

21. Colombier, J. P. et al. Optimized energy coupling at ultrafast laser-irradiated metal surfaces by tailoring intensity envelopes: Consequences for material removal from Al samples. Phys. Rev. B 74, 224106 (2006).

22. Kahaly, S. et al. Direct observation of density gradient effects in harmonic generation from plasma mirrors. Phys. Rev. Lett. 110, 175001 (2013).

23. Mairesse, Y. \& Quéré, F. Frequency-resolved optical gating for complete reconstruction of attosecond bursts. Phys. Rev. A 71, 011401 (2005).

24. Sansone, G. et al. Isolated single-cycle attosecond pulses. Science $\mathbf{3 1 4}$, 443-446 (2006). 
25. Kammel, S. \& Leon, F. Deflectometric measurement of specular surfaces. IEEE Trans. Instrum. Meas. 57, 763-769 (2008).

26. Dromey, B. et al. Diffraction-limited performance and focusing of high harmonics from relativistic plasmas. Nature Phys. 5, 146-152 (2009).

27. Quéré, F. et al. Coherent wake emission of high-order harmonics from overdense plasmas. Phys. Rev. Lett. 96, 125004 (2006).

28. Quéré, F. et al. Phase properties of laser high-order harmonics generated on plasma mirrors. Phys. Rev. Lett. 100, 095004 (2008).

29. Ceccotti, T. et al. Evidence of resonant surface-wave excitation in the relativistic regime through measurements of proton acceleration from grating targets. Phys. Rev. Lett. 111, 185001 (2013).

30. Kahaly, S. et al. Near-complete absorption of intense, ultrashort laser light by sub- $\lambda$ gratings. Phys. Rev. Lett. 101, 145001 (2008)

31. Purvis, M. A. et al. Relativistic plasma nanophotonics for ultrahigh energy density physics. Nature Photon. 7, 796-800 (2013).

32. Fedeli, L. et al. Electron acceleration by relativistic surface plasmons in laser-grating interaction. Phys. Rev. Lett. (in the press)

33. Spangenberg, D., Neethling, P., Rohwer, E., Brügmann, M. H. \& Feurer, T. Time-domain ptychography. Phys. Rev. A 91, 021803 (2015).

34. Kim, K. T. et al. Manipulation of quantum paths for space-time characterization of attosecond pulses. Nature Phys. 9, 159-163 (2013).

\section{Acknowledgements}

We are grateful to P. d'Oliveira, F. Réau, C. Pothier and D. Garzella for operating the UHI100 laser source. The research leading to these results has received financial support from the European Research Council (ERC Grant Agreement number 240013), from Investissements dAvenir LabEx PALM (ANR-10-LABX-0039-PALM) through the 'Ptychograt' grant, from Agence Nationale pour la Recherche through grant ANR-14-CE32-0011, from OSEO through SAPHIR (contact number I0901001W), and from LASERLAB-EUROPE (grant agreement no. 284464, EC’s Seventh Framework Programme) through the INREX Joint Research Action.

\section{Author contributions}

F.Q. proposed the idea of the measurement scheme. S.M., A.L., S.K. and F.Q. conceived the experimental set-up. The experiment was carried out by A.L. and S.M. with the help of S.K. A.L. processed the experimental data, C.B.-B. wrote the phase-retrieval program, A.L. and C.B.-B. worked together on the application of this program to the experimental results. The overall work was supervised by F.Q.

\section{Additional information}

Supplementary information is available in the online version of the paper. Reprints and permissions information is available online at www.nature.com/reprints.

Correspondence and requests for materials should be addressed to F.Q.

\section{Competing financial interests}

The authors declare no competing financial interests. 